\title{
Quantum Dot Cytotoxicity and Ways To Reduce It
}

\author{
FRANÇOISE M. WINNIK*, ${ }^{*} \neq$ AND DUSICA MAYSINGER ${ }^{\S}$ \\ ${ }^{\dagger}$ Faculty of Pharmacy and Department of Chemistry, University of Montreal, \\ CP 6128 Succursale Centre Ville, Montreal QC H3C 3J7, Canada, ${ }^{\ddagger}$ International \\ Center for Materials Nanoarchitectonics (WPI-MANA), National Institute for \\ Materials Science (NIMS), Tsukuba, Ibaraki 305-0044, Japan, and \\ ${ }^{\S}$ Department of Pharmacology \& Therapeutics, McGill University, \\ Montreal QC H3G 1Y6, Canada
}

RECEIVED ON FEBRUARY 22, 2012

\section{CONSPECTUS}

$\mathrm{T}$ he dramatic increase in the use of nanoparticles (NP) in industry and research has raised questions about the potential toxicity of such materials. Unfortunately, not enough is known about how the novel, technologically-attractive properties of NPs correlate with the interactions that may take place at the nano/bio interface. The academic, industrial, and regulatory communities are actively seeking answers to the growing concerns on the impact of nanotechnology on humans. In this Account we adopt quantum dots (QDs) as an illustrative example of the difficulties associated with the development of a rational science-

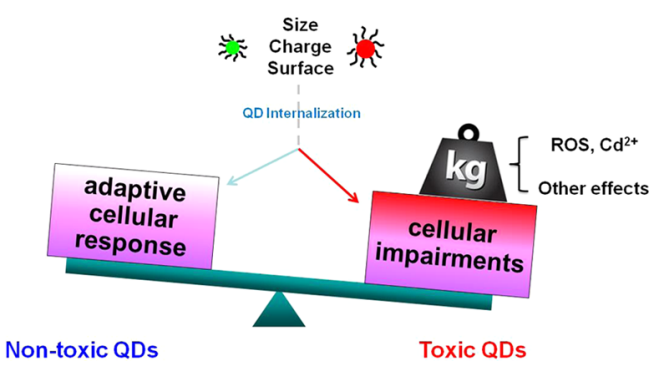
based approach to nanotoxicology.

The optical properties of QDs are far superior to those of organic dyes in terms of emission and absorption bandwidths, quantum yield, and resistance to photobleaching. Moreover, QDs may be decorated with targeting moieties or drugs and, therefore, are candidates for site-specific medical imaging and for drug delivery, for example in cancer treatment. Earlier this year researchers demonstrated that QD-based imaging using monkeys caused no adverse effects although QDs accumulated in lymph nodes, bone marrow, liver, and spleen for up to 3 months after injection. Such persistence of QDs in live animals does, however, raise concerns about the safety of using QDs both in the laboratory and in the clinic.

Researchers anticipate that QDs will be increasingly used not only in clinical applications but also in various manufactured products. For example, QD-solar cells have emerged as viable contenders to complement or replace dye-sensitized solar cells; $\mathrm{CdTe} / \mathrm{CdS}$ thin film cells have already captured approximately 10 percent of the global market, and in addition, QDs can serve as components of sensors and as emitting materials in LEDs. Given the clear indications that QDs will inevitably become components of a wide range of manufactured and consumer products, researchers and policy makers need to understand the possible health risks associated with exposure to QDs.

In this Account, we initially review the known mechanisms by which QDs can damage cells, including oxidative stress elicited by reactive oxygen species (ROS). We discuss lesser-known impairments induced in cells by nanomolar to picomolar concentrations of QDs, which imply that cadmium-containing QDs can exert genotoxic, epigenetic, and metalloestrogenic effects. These observations strongly suggest that minute concentrations of QDs could be sufficient to cause long lasting, even transgenerational, effects. We also consider various modes by which humans could be exposed to QDs in their work or through the environment. Although considerable advances have been made in enhancing the stability and overall quality of QDs, over time they can partially degrade in the environment or in biological systems, and eventually cause small, but cumulative undesirable effects.

A combination of toxicological, genetic, epigenetic and imaging approaches is required to create comprehensive guidelines for evaluating the nanotoxicity of nanomaterials, including QDs. Prior to biological investigations with these materials, an indispensible step must be the full characterization of NPs by complementary techniques. Specifically, the concentration, size, charge, and ligand stability of NPs in biological media must be known if we are to understand fully how the properties of nanoparticles and of their biological environment contribute to cytotoxicity. 


\section{Introduction}

Real-time in vitro and in vivo imaging of cellular mechanisms using quantum dots (QDs) has become a major tool in biology and in exploratory nanomedicine due to the versatility and exceptional photostability of QDs, compared to organic dyes. ${ }^{1}$ Early on, biologists noted that QDs were not necessarily as innocuous to cells as soluble dyes, as a consequence of their particulate nature and composition. ${ }^{2,3}$ In the early 2000s, we became aware that QDs may exert adverse effects in biological systems. ${ }^{4,5}$ As we exposed different cell types to CdTe QDs, we noted a number of changes in cell morphology. Results from studies employing different QD concentrations, different exposure times, and different cell types clearly indicated that (i) cadmium-containing QDs, most prominently CdTe QDs, were the most deleterious to the cells, particularly QDs with short ligands; (ii) QDs with multiple shells, e.g. CdSe/ZnS QDs, were suitable for experiments with living cells exposed for longer time periods (hours and even days) without significant functional deteriorations; and (iii) the complexity of the cellular system (monolayers vs 3D-mixed cell populations) played an additional role in determining cell susceptibility to the QDs: generally cells in monolayers were the most vulnerable to the nanocrystals.

To account for the cellular impairments inflicted by CdTe QDs, we hypothesized that the QDs may have been contaminated with soluble $\mathrm{Cd}^{2+}$ or that $\mathrm{Cd}^{2+}$ was released from the QDs after internalization in cells. There is no lack of data on the toxicity of cadmium, ${ }^{6}$ which has been associated with liver and kidney injuries, skeletal deformations, neurological problems, and cancer. On the cellular level, cadmium induces the formation of reactive oxygen species (ROS) by depletion of cellular antioxidants. It affects mitochondria, induces apoptosis, disrupts intracellular calcium signaling, damages DNA, and inhibits DNA repair. Most of these adverse effects are also characteristic of QD-induced cellular toxicity, so it is reasonable to conclude that QDs are toxic because, as a result of imperfections in the nanoparticles coating, $\mathrm{Cd}^{2+}$ is gradually released in cells after internalization of QDs. The fact that CdSe/ZnS QDs are significantly less toxic than "bare" CdTe QDs also argues in favor of this conclusion. A corollary, of course, is that chemists can address, and solve, the toxicity issues related to Cd-based QDs by covering the $\mathrm{Cd}$ core by a robust outer layer, impervious to the QD environment.

We were concerned that the situation may not be so simple. Could QDs not be toxic per-se, by virtue of their size and photophysical properties? Composition, after all, is just one aspect of nanoparticle toxicity. Particle size influences their behavior in cells: smaller particles tend to be more toxic than larger ones. ${ }^{4}$ Of course, as particle size decreases, the surface to volume ratio increases; hence, more $\mathrm{Cd}^{2+}$ ions are exposed and available for poisoning their environment. But, it is possible also that specific cellular pathways are open only to smaller particles, directing them to selected cell organelles or opening access to the nucleus. There is no denying that ROS can be generated from the QD surface. One can expect that the (size dependent) redox properties of nanoparticles affect the amount of ROS produced. Photoirradiation of QDs provides another means to generate free radicals, a process evidently affected by the quantum size effect in the core. These considerations make it increasingly difficult to argue that $\mathrm{Cd}^{2+}$ is responsible for all aspects of QD toxicity.

Conceptually, one would expect that if $\mathrm{Cd}^{2+}$ is indeed solely responsible for cellular impairment and death, then cell viability should be inversely proportional to the intracellular $\mathrm{Cd}^{2+}$ concentration $\left[\mathrm{Cd}^{2+}\right]_{\text {intra. }}$. Both quantities can be determined experimentally, and we measured them for human breast cancer cells (MCF-7) exposed to several samples of CdTe QDs and CdSe/ZnS QDs. ${ }^{7}$ Cell viability assays indicated that CdSe/ZnS QDs were nontoxic to cells whereas CdTe QDs were toxic and cell viability was proportional to the initial QD dose. From confocal fluorescence microscopy imaging, we knew that no observable injury was detected in cells treated with CdSe/ZnS QDs, whereas CdTe QDs brought about serious damaging changes in cells. Prominent changes included lysosomal enlargement and mitochondrial rounding, two features exhibited by cells under oxidative stress. We established that, for cells treated with solutions of $\mathrm{CdCl}_{2}$ (not QDs), the decrease in cell viability correlates linearly with $\left[\mathrm{Cd}^{2+}\right]_{\text {intra }}$ (Figure 1). Next, we measured $\left[\mathrm{Cd}^{2+}\right]_{\text {intra }}$ in cells treated with CdSe/ZnS QDs; this concentration was $<5 \mathrm{nM}$. In cells treated with CdTe QDs, $\left[\mathrm{Cd}^{2}\right]_{\text {intra }}$ ranged from 30 to $150 \mathrm{nM}$, depending on the nature of the ligand. However, the $\left[\mathrm{Cd}^{2+}\right]_{\text {intra }}$ did not correlate linearly with cell viability (Figure 1). Hence, the toxic effect of CdTe QDs cannot be attributed solely to the release of $\mathrm{Cd}^{2+}$. The two facets of QDs toxicity, which are clearly exhibited by CdTe QDs, cannot be overlooked, in particular when considering applications in nanomedicine or when assessing the environmental impact of QDs.

\section{Known (and Suspected) Insults by QDs to Cells and to Organisms}

We present below an overview of the major routes by which QDs can injure cells and organisms. The adverse effects 


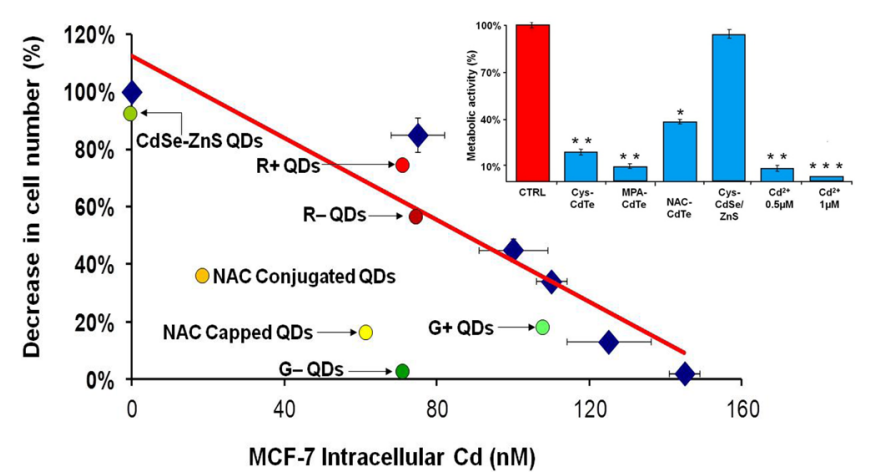

FIGURE 1. Decrease in cell number as a function of $\left[\mathrm{Cd}^{2+}\right]_{\text {intra }}$ for MCF-7 cells treated with various QD samples $(10 \mu \mathrm{g} / \mathrm{mL})$; inset: metabolic activity of MCF-7 cells treated with QDs or with $\mathrm{CdCl}_{2}$ aqueous solutions. Adapted from Figures 1 and 5 of ref 7. Copyright 2007 American Chemical Society.

are organized from the point of view of the affected cells and the mechanisms known (or suggested) to be involved in the cellular response. Figure 2 presents an overview of the cellular mechanisms (discussed below) that can be triggered by QD internalization together with representative micrographs of impaired cells. We have balanced the toxicity studies carried out using CdSe/ZnS QDs, the standard QDs in biological studies, and other types of QDs, in order to lead the reader to the conclusion that materials combinations, size ranges, and surface capping materials really matter with respect of QDs toxicity in vitro and in vivo. ${ }^{3,6,8}$ This point was demonstrated most recently in systematic studies by Chan's group conducted in vivo using different core-corona nanomaterials that convincingly show that QD composition ( $\mathrm{PbS}$ vs CdS), size ( $2 \mathrm{~nm}$ vs $6 \mathrm{~nm}$ ), shape (spheres vs rods), and surface chemistry (amino vs carboxylic group) play an important role in biodistribution and toxicity. ${ }^{9}$ Similarly, a systematic study by Rotello's team ${ }^{10}$ showed that the stability of the monolayer surface coating of QDs depends both on the quantum dot particle size and on the monolayer chemical structure. Their studies using label free mass spectrometry determinations show that intracellular stability can be markedly improved by selecting the appropriate QD size and surface ligand.

2.1. QDs as Disruptors of Cellular Redox Homeostasis. An increased ROS production results in an oxidative stress when cells fail to compensate for the increased [ROS] and consequently fail to maintain or restore normal physiological redox-regulated functions. Several compensatory mechanisms are put in motion when redox equilibrium is disrupted in order to prevent cellular impairments, which could ultimately lead to cell death. ${ }^{11}$ The cellular response is incremental, depending on the ROS production level. Under normal conditions, very low amounts of ROS are generated in cells. As soon as cells detect ROS, "alarm signals" are sent, triggering antioxidant defenses, such as glutathione and antioxidant enzymes (Figure 3). This "adaptation phase" usually restores the reducing environment of the cell. However if excess ROS generation persists, the natural defense mechanisms of the cell are overwhelmed, leading to oxidative stress, ultimately leading to cell death (e.g., apoptosis, necrosis, or autophagy). The concept of hierarchical oxidative stress has been exploited by Nel to devise an integrated set of cellular screening assays of nanoparticles that quantify specific cellular responses to each level of oxidative stress. ${ }^{12}$

ROS and the Vasculature. The cells that line the luminal surface of all blood vessels, unavoidably, are exposed to QDs circulating in the bloodstream following intravenous injection, the most common administration mode for in vivo imaging/therapeutic applications. Vascular endothelial cells are especially susceptible to ROS-induced mitochondrion damage that may promote atherosclerosis and cardiovascular diseases. ${ }^{13}$ Yan et al. tested the toxicity of acid coated CdTe QDs toward human umbilical vein endothelial cells (HUVEC), which are models for vascular endothelial cells. ${ }^{14}$ QD concentrations in the $0.1-100 \mu \mathrm{g} / \mathrm{L}$ range were selected, since they correspond to the doses commonly used for in vivo imaging. Exposure of HUVEC to CdTe QDs for $24 \mathrm{~h}$ induced an intrinsic, i.e. mitochondria-mediated, apoptosis.

ROS and the Skin and the Eye. Dermal cells constitute the natural barrier against penetration of QDs through the skin, for example while handling QDs or in case of manufacturer exposure. In vitro studies have demonstrated that QDs, in the micromolar concentration range, can penetrate the epidermis to reach the dermis, particularly via the hair follicles or through stretched skin. ${ }^{15,16}$ More recent studies have assessed the effects of nanomolar QD concentrations, which were shown to lead to DNA perturbations, thereby leading to QD-induced abnormalities at the genomic level. ${ }^{17}$ Kuo et al assessed the consequence to the eye of exposure to CdSe/ZnS QDs coated with PEGs, amines, or carboxylates $(\sim 20 \mathrm{nM}) .{ }^{18}$ QDs administered intrastromally in mice were retained in the eye for up to 26 days. Complementary in vitro studies with bovine corneal stromal cells indicated that cell viability decreased significantly upon prolonged exposure (48 h) to QDs, particularly with carboxylated QDs.

QDs Effects on the Nervous System. Our early in vitro 4 and in vivo studies showed rather marked responses of astrocytes ${ }^{19}$ and microglia ${ }^{20}$ to several types of QDs. The majority of intraparenchimally injected QDs were localized within microglial cells, which normally function as the brain 


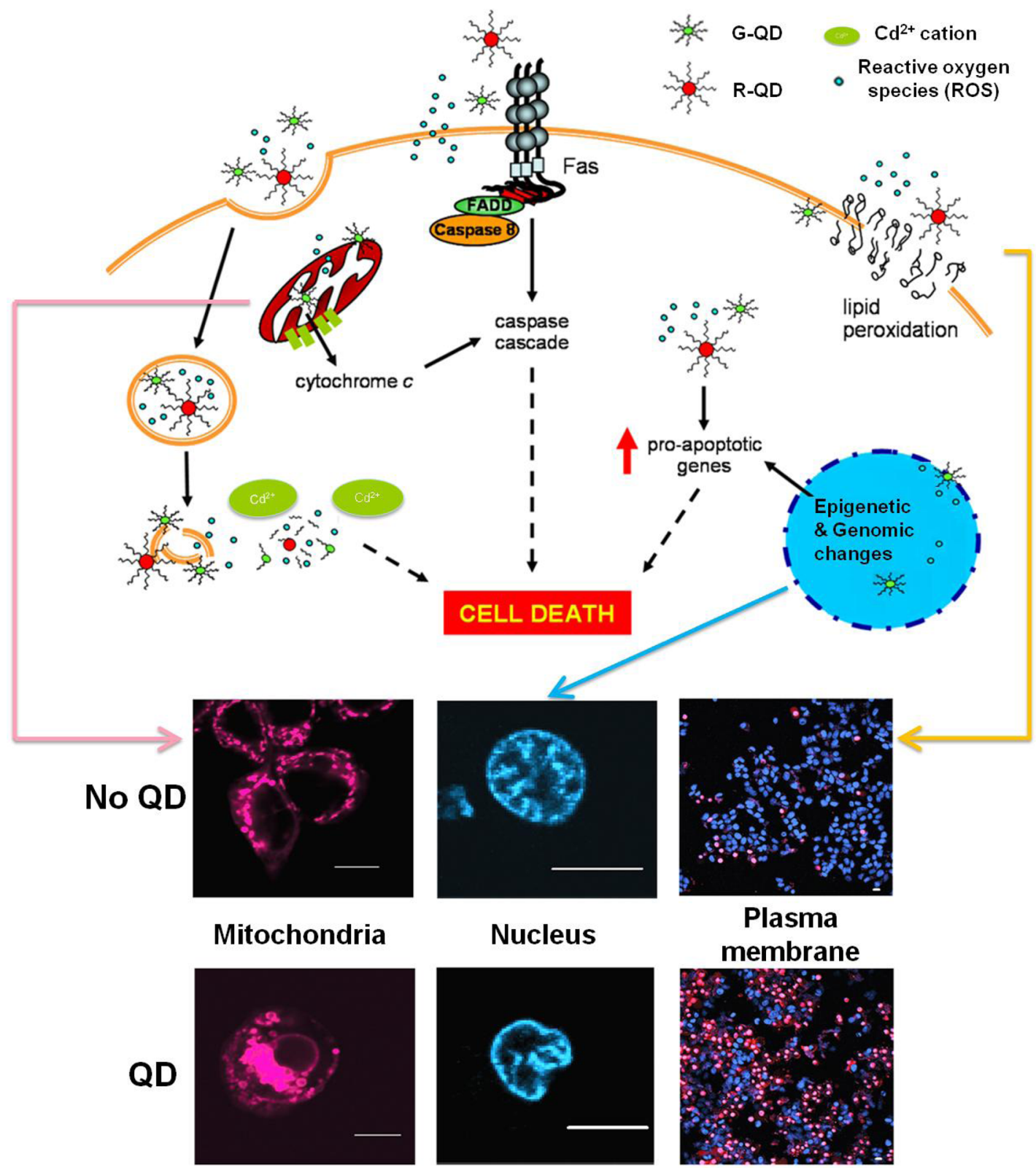

FIGURE 2. Top: Schematic overview of the cellular mechanisms shown to occur in cells treated with the Cd-containing QDs discussed in section 3. Bottom: Examples of QD-triggered impairments of the mitochondrion, nucleus, and plasma membrane. Images reproduced and adapted from Figure 1 in ref 5. Copyright 2005 Elsevier.

phagocytes. While upon exposure of the brain to PEGylated CdSe QDs the activation of astrocytes in the cerebral cortex was detected only transiently, the administration of nonPEGylated CdTe QDs triggered a robust glial activation without significant cell loss. Tang et al. showed that injections of either CdSe or streptavidin-CdSe/ZnS QDs (0.5 nM) into the hippocampal dentate gyrus area of rats led to impaired short- and long-term synaptic activity. ${ }^{21}$

More recently, CdSe/ZnS QDs were shown to increase intracellular $\mathrm{Ca}^{2+}$ in rat hippocampal neurons ${ }^{22}$ and in mouse chromaffin cells. ${ }^{23}$ Studies of renal epithelial cells exposed to QDs have revealed that F-actin binds to QDs and can be depolymerized by $\mathrm{Cd}^{2+}$ ions. ${ }^{24}$ Since F-actin is postulated to play a role in controlling secretory granule access to the plasma membrane, it is possible that the reduced neurosecretion may be due to F-actin impairment by QDs and/or released $\mathrm{Cd}^{2+}$.

2.2. Metalloestrogenic Effects of QDs. A lesser known property of cadmium is that, on the picomolar level, it can exert estrogenic effects. ${ }^{25}$ Could it be possible that very low concentrations of Cd-containing QDs have corresponding estrogenic effects? We examined this issue by monitoring 
the response of MCF-7 cells to CdTe QDs in concentrations not displaying significant nonestrogenic cytotoxicity. ${ }^{26} \mathrm{CdTe}$ QDs induced cellular proliferation, ER $\alpha$ (estradiol receptor $\alpha$ ) activation, and estrogen-associated rapid nongenomic signaling events, comparable to, or even exceeding, those caused by $17 \beta$-estradiol. Green (smaller) QDs elicited a more robust estrogenic effect than orange (larger) QDs (Figure 4). The QD estrogenic effects were completely suppressed in cells supplemented with the selective estrogen receptor antagonist ICl 182780, indicating that the QD-induced estrogenic signaling is mediated by the estrogen receptor. MCF-7 cells express the estrogen receptors $\operatorname{ER} \alpha$ and $\operatorname{ER} \beta$ and the plasma membrane estrogenic receptor GPR30.

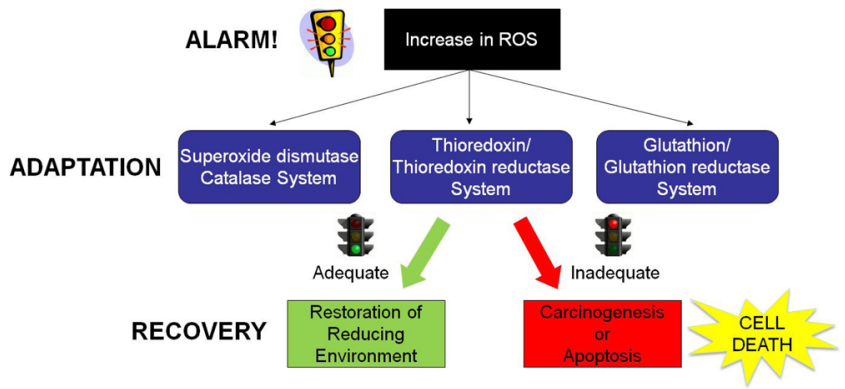

FIGURE 3. Pictorial representation of the hierarchical response of cells to oxidative stress.
QD-estrogenicity in vivo in black BalbC57 mice was detected after intraperitoneal injections of CdTe QDs and compared with $17 \beta$-estradiol $(0.25 \mathrm{mg} / \mathrm{kg})$ and $\mathrm{Cd}^{2+}$ $(0.25 \mathrm{mg} / \mathrm{kg}){ }^{26}$ The doses of $17 \beta$-estradiol and $\mathrm{Cd}^{2+}$ were those reported to elicit maximal estrogenic response. Green QDs elicited the most potent estrogenic effects in both prepubescent animals and in older, ovariectomized animals. This study suggests that cadmium-containing QDs should be considered as potential endocrine disruptors, causing an increase of the overall estrogenic burden in cells and tissue and, possibly, leading to carcinogenicity. In vitro and in vivo studies are warranted in order to delineate signal transduction pathways mediating metallo-estrogenic effects caused by suspect endocrine disruptor QDs and to establish likely epigenomic influences modulating the estrogen-like effects.

2.3. Nanogenomic Effects of QDs. By definition, genotoxic agents damage DNA, with resulting loss of DNA integrity, mutagenesis, and chromosomal aberrations. Nanoparticles (NP) as a group are known to induce genotoxic effects by a variety of mechanisms, ${ }^{27}$ including (1) direct interaction with DNA in the case of NPs (such as small QDs) able to enter the nucleus; (2) impairment of the cellular transcription and translation machinery as a consequence of the proximal perinuclear localization favored by QDloaded lysosomes; (3) interactions with surface receptors,
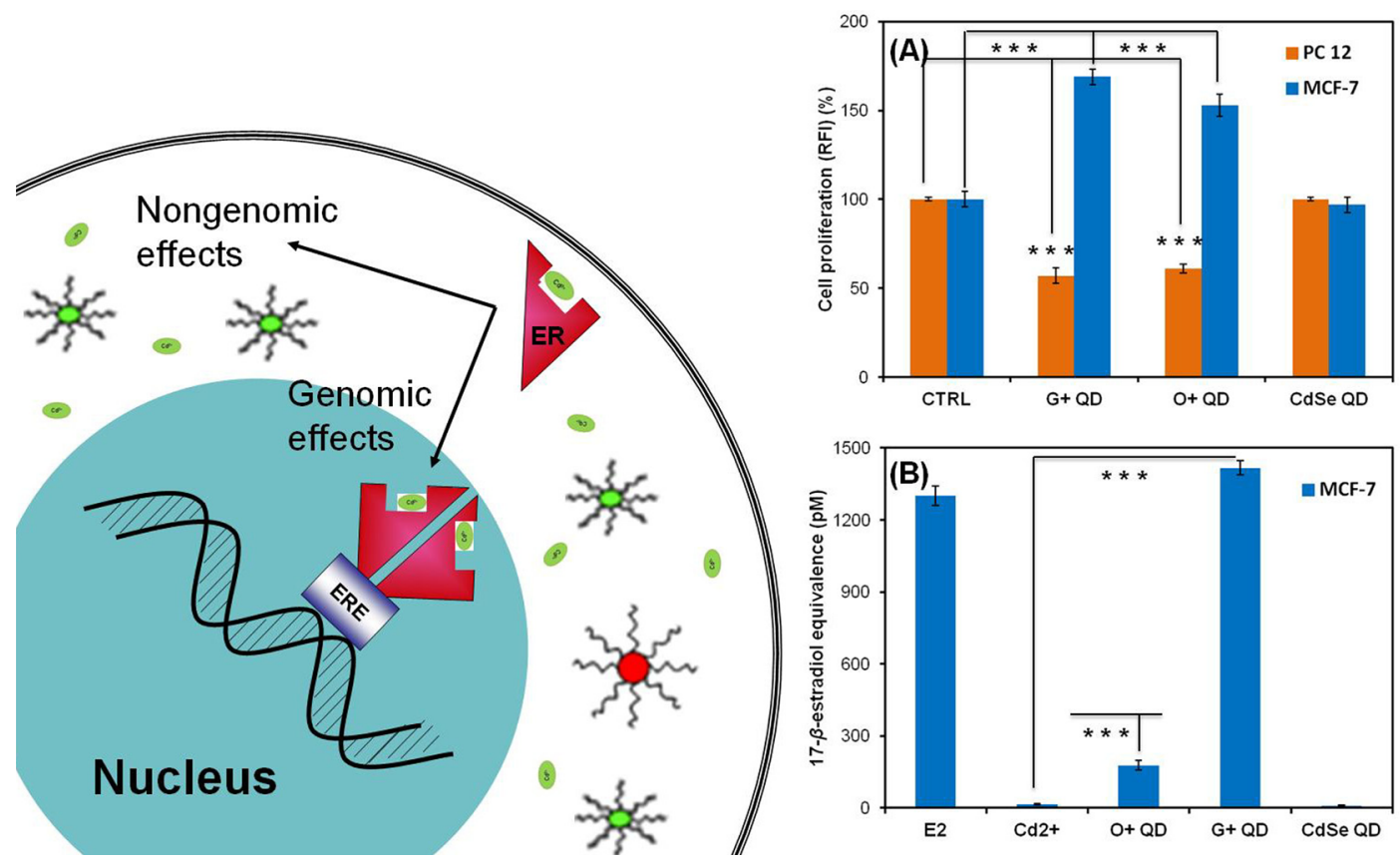

FIGURE 4. QD induced metalloestrogenicity. Surface compromised CdTe QDs can leach $\mathrm{Cd}^{2+}$, either extracellularly leading to $\mathrm{Cd}^{2+}$ internalization via transporters or after internalization and degradation within the cell. Released $\mathrm{Cd}^{2+}$ may trigger estrogenicity via genomic and/or nongenomic effects: (A) cell proliferation induced by green $\left(\mathrm{G}^{+}\right)$and orange $\left(\mathrm{O}^{+}\right)$CdTe QDs $(10 \mu \mathrm{g} / \mathrm{mL})$ in ER-expressing (MCF-7) and non-ER-expressing (PC-12) cells; (B) estradiol $\left(E_{2}\right)$ equivalence of $C^{2+}$ and QDs after 24-h treatment of MCF-7 cells with estrogen. Adapted from Figures 1 and 4 of ref 26. Copyright 2012 Rightlinks. 
resulting in receptor activation and enablement of intracellular signaling cascades (see metalloestrogenic effects of QDs); and (4) inducement of DNA point mutations or/and single- or double-strand breaks via ROS generated by NPs localized in the lysosomes.

Addition of QDs to DNA solutions is known to lead to DNA damage upon irradiation with light of $\lambda>310 \mathrm{~nm}$, a spectral region where DNA itself does not absorb, ${ }^{28}$ or, in the dark, via ROS mediated chemical reactions. ${ }^{29}$ Hoshino et al. were among the first to report the detection of DNA impairments inflicted by CdSe/ZnS QDs to mammalian cells. ${ }^{30}$ Other groups suggested DNA damage by QDs, on the basis of changes in nuclear morphology and from up- or downregulation of specific proteins. The effects of QD exposure to gene expression itself were monitored in a study involving treatments of a human dermal fibroblast (HDF) cell line with CdSe/ZnS QDs (30-60 nM, diameter $\sim 15 \mathrm{~nm}$ ). ${ }^{17}$ Cells exposed to such QDs modulated genes and proteins indicative of oxidative stress, apoptosis, inflammation, and more general immune responses point toward the need to study mechanisms of actions triggered by QDs among different cell types of the same organ, both in vitro (preferably in human cells) and in vivo.

Gagné et al. analyzed hepatic gene expression in rainbow trout exposed to either CdS/CdTe QDs or (soluble) $\mathrm{Cd}^{2+}$ using a DNA microarray comprised of 207 stress-related genes. ${ }^{31}$ On a mass concentration basis, QDs were more potent than $\mathrm{Cd}^{2+}$ in suppressing immunocompetence. Both forms of cadmium induced expression of the metallothionein and CP2K1 genes involved, respectively, in metal detoxification and xenobiotic transformation/inflammation conditions. However, the response pattern was different for the two $\mathrm{Cd}$ forms. Exposure to QDs significantly affected 25 genes that are mainly implicated in oxidative stress and inflammation. Only 9 genes were affected by exposure to $\mathrm{Cd}^{2+}$. The impaired genes in this case are implicated in the binding and transport of solutes, such as urea, sodium, potassium, etc., or in aspects of the immune status different from those altered as a consequence of QD exposure.

2.4. Nanoepigenomic Effects of QDs. Epigenetic agents modify the expression of genetic information, without altering the primary DNA sequences. ${ }^{32}$ The alterations of genetic information may eventually lead to a long-term reprogramming of gene expression. Epigenetic programming typically occurs during development. However, an increasing number of studies suggest that the adult epigenome is sensitive to environmental factors. ${ }^{33}$ Proposed epigenetic cellular mechanisms include the following: (1) modifications of the

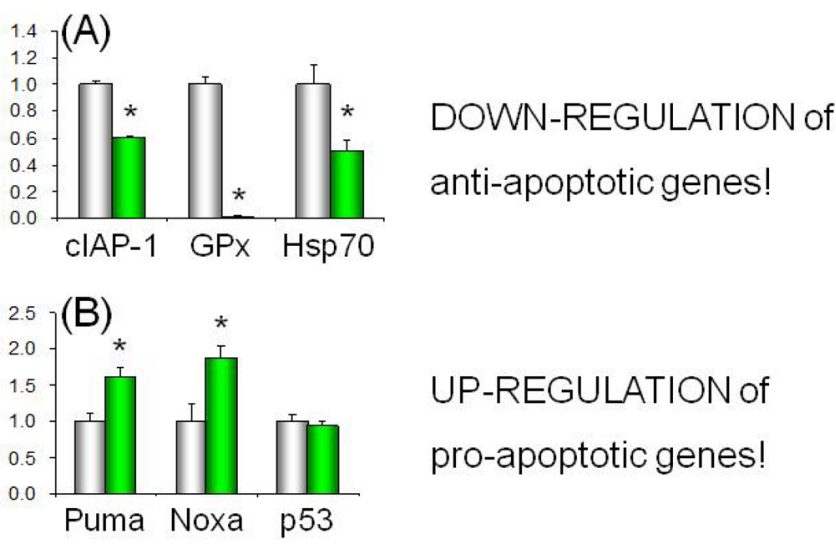

FIGURE 5. Changes in mRNA levels of (A) antiapoptotic and (B) proapopoptotic genes in MCF-7 cells treated with CdTe QDs (5 $\mu \mathrm{g} / \mathrm{mL})$ in the absence or presence of trichostatin (TSA) and control. Adapted from Figure 3 of ref 34. Copyright 2008 Springer.

chromatin and histone structures, such that access of the transcriptional machinery to genes is prevented; (2) inhibition of translation and degradation of RNA; and (3) damaging effects on DNA itself, since DNA bears epigenetic information in its methylation pattern. Epigenetic changes may have long-term effects on gene expression programming, long after the initial signal has been removed and, if these changes remain undetected, this situation could lead to long-term untoward effects in biological systems.

Small green CdTe QDs can enter the nucleus and cause significant reorganization of the chromatin, which strongly suggests that gene transcription is affected. Chromatin reorganization was detected upon exposure of MCF-7 cells to low amounts $(5 \mu \mathrm{g} / \mathrm{mL})$ of green CdTe QDs for $24 \mathrm{~h}$. It was accompanied by a significant change in global hypoacetylation, implying an epigenomic response. ${ }^{34}$ The magnitude of the reduction in histone acetylation was inversely proportional to [QD], such that the higher the [QD], the more histone acetylation decreased. These epigenetic changes corresponded with (i) the reduction in the transcription of genes associated with cell death prevention, such as CIAP-1 (inhibitor of apoptosis) and Hsp70 (heat shock protein 70), and the suppression of GPx (glutathione peroxidase) mRNA expression and (ii) the activation of the ubiquitous responder to genotoxic stress, p53, resulting in translocation of p53 with subsequent upregulation of the downstream targets Puma and Noxa (Figure 5). QD-induced epigenetic changes along with the genotoxic effects merit thorough examination, not only in model cells but also in living organisms. This study strongly advocates in favor of the inclusion of epigenomic assays in the battery of tests routinely used in nanoparticles toxicological studies. 


\section{Known (and Anticipated) Means of Exposure to Quantum Dots}

Unlike silver or titanium dioxide NPs, which can be found in many consumer products, QDs have yet to reach the mass market. This situation may change rapidly. A recent review on CdTe QDs lists major achievements toward their use in the fabrication of all inorganic photovoltaic devices, solar cells, and sensors or as emitting materials in light emitting diodes etc. ${ }^{35}$ Should QDs become parts of consumer products, what are the potential routes of exposure by which humans could be exposed to QDs, at least in theory?

Aerosolization and accidental inhalation of QDs could occur during their manufacture or potentially as a means of clinical administration. On the basis of toxicological studies of airborne NPs, one can envisage that retention of QDs in the lungs may occur, potentially with translocation in the central nervous system. ${ }^{38}$ Inadvertent adsorption through the skin and eyes could occur during QD manufacture or QD use in the laboratory and, possibly, in the clinic. Model studies indicate that QDs are able to penetrate porcine skin and cause impairing effects, even in low doses. ${ }^{15-17}$ The cornea is another route of drug administration and an important route of exposure in occupational situations and in daily life. The observation that QDs can penetrate injured cornea and be retained in it asks for further studies, since stromal cells are important to ensure the health and transparency of the cornea. Corneal abrasion is not uncommon in our daily life, particularly among wearers of contact lenses or as a consequence of the application of cosmetics or UVblocking agents spiked with nanoparticles.

Oral ingestion for therapeutic or imaging purposes is not envisaged currently, since there are concerns on the limited stability of QDs in the acidic gastric milieu. Nonetheless, insidious QD ingestion could occur by transfer of QDs through the food chain. Gagné et al. reported that CdTe QDs are ingested by freshwater mussels ${ }^{36}$ and by rainbow trout. ${ }^{30}$ Also, CdTe and CdSe QDs were shown to accumulate in E. coli cells ${ }^{37}$ and in Pseudomonas aeruginosa bacteria, ${ }^{38}$ respectively. Moreover, Werlin et al. reported that red citrate-capped CdSe QDs accumulated in $P$. aeruginosa can be transferred and biomagnified in Tetrahymena thermophilia protozoae that prey on the bacteria. ${ }^{39}$ The Cd-loaded bacteria inhibited their own digestion in the protozoan food vacuoles, where they remained without being lysed. Hence, intact QDs remained available in higher trophic levels, resulting in cadmium concentrations in the protozoae $\sim 5$ times higher than in their prey. This study provides the first example of biomagnification of QDs in a model microbial food chain. The authors of this study point out that, to ensure scientific reliability, unrealistically high QD concentrations were used and added in the batch mode. Corresponding measurements with a steady influx of a low concentration of QDs are necessary to mimic conditions within the ecological environment.

Finally, one needs to cite the recent report by Chu et al., who presented evidence that core/shell CdTe/CdS QDs can be transferred from female mice to their fetuses across the placental barrier. ${ }^{40}$ Smaller QDs were more readily transferred than larger ones. PEGylation of QDs reduced the transfer but did not eliminate it, suggesting the need to establish the consequences of long-term exposure to picomolar concentrations of QDs. Because Cd-containing QDs are candidates for trans-generational effects, their use should be minimized; if used, they should be handled with caution.

\section{Conclusions and Outlook}

Undesirable effects caused by early generations of cadmium-containing QDs are likely to be reduced by new generations of QDs. The quest for novel nanoparticles as effective as current QDs, but devoid of proven toxic elements, has intensified, and new highly promising luminescent NPs have been discovered over the past few years. Luminescent carbon nanodots (C-dots), ${ }^{41}$ graphene quantum dots (GQDs), and nanometer-size graphene pieces with excellent optical and electronic properties were recently described. ${ }^{42}$ These NPs offer much promise in view of their chemical inertness, high surface area, and good surface grafting capacity. ${ }^{43}$ Preliminary data indicate that both C-dots $^{44}$ and GQDs ${ }^{45}$ exhibit minimal cytotoxicity to exposed cells. Silicon QDs as well offer a promising alternative to semiconductor QDs, although their photoluminescence quantum yields (5-10\%) are lower than those of CdSe QDs. They exhibit minimal cytotoxicity ${ }^{46}$ and were used successfully for in vitro ${ }^{47}$ and in vivo ${ }^{48}$ imaging.

The wealth of data accumulated from the toxicity of semiconductor NPs is an invaluable asset that should be exploited to design appropriate methodologies to assess the toxicity of novel luminescent NPs. Researchers often neglect to carry out a comprehensive characterization of QDs prior to using them. In our opinion, this step is imperative, ${ }^{49}$ especially before any toxicity screening is started, precisely because the exact property (or properties) of QDs responsible for their toxicity is still poorly understood. This omission is one of the reasons behind the current state 
of confusion surrounding the question of QD toxicity. ${ }^{50}$ As epigenetic changes may lead to long-term reprogramming of gene expression long after the initial insult has been removed, results from "nanoepigenetic" assessments may have important implications on the future use of new nanomaterials in bioimaging and therapeutic applications and should be evaluated early in the development of new QDs, as well as QD-based devices and clinical tools. It would be useful also to devise tests to assess if subtle epigenetic changes could be detected in blood samples of humans chronically exposed to minute concentrations of nanomaterials in their working environment (e.g., factories producing solar cells or certain types of electronics).

This work was funded in part by the Natural Sciences and Engineering Research Council of Canada (F.M.W. and D.M.) and in part by the World Premier International Research Center Initiative (WPI) MEXT Japan (FMW). We are grateful to current and former collaborators and members of our research groups for their scientific contributions and enthusiasm. Additional thanks go to A. Moquin and Y. T. R. Lau for valuable editorial help.

\section{BIOGRAPHICAL INFORMATION}

Françoise M. Winnik obtained her Ph.D. from the University of Toronto. She worked as a research scientist in the Xerox Research Center of Canada, before joining McMaster U (Hamilton ON) in 1993 as an Associate Professor. Since 2000, she has been a professor in the Université de Montréal. Her research interests include self-assembly of amphiphilic polymers, nanoparticles, and biointerfaces.

Dusica Maysinger earned her Ph.D. at the University of Southern California, Los Angeles and pursued her postdoctoral training at the Max Planck Institute, Karolinska Institute, and Oxford University. Her research activities focus on understanding cell/nanomaterial interactions and the subsequent activation of cellular transduction pathways.

\section{FOOTNOTES}

*Corresponding author. E-mail: francoise.winnik@umontreal.ca. Tel: 1-514-340-5179.

The authors declare no competing financial interest.

\section{REFERENCES}

1 For an up-to-date ovenview, see: Mattoussi, H.; Palui, G.; Na, H. B.: Luminescent quantum dots as platforms for probing in vitro and in vivo biological processes. Adv. Drug Delivery Rev. 2011, DOl: 10.1016/j.addr.2011.09.011 and references therein.

2 Hardman, R. A toxicologic review of quantum dots: Toxicity depends on physicochemical and environmental factors. Environm. Health Perspect. 2006, 114, 165-172.

3 Lewinski, N.; Colvin, V.; Drezek, R. Cytotoxicity of nanoparticles. Small 2008, 4, 26-49.

4 Lovric, J.; Bazzi, H. S.; Cuie, Y.; Fortin, G. R.; Winnik, F. M.; Maysinger, D. Differences in subcellular distribution and toxicity of green and red emitting CdTe quantum dots. J. Mol. Med. 2005, 83, 377-385.
5 Lovric, J.; Cho, S. J.; Winnik, F. M.; Maysinger, D. Unmodified cadmium telluride quantum dots induce reactive oxygen species formation leading to multiple organelle damage and cell death. Chem. Biol. 2005, 12, 1227-1234.

6 Rzigalinski, B. A.; Strobl, J. S. Cadmium-containing nanoparticles: Perspectives on pharmacology and toxicology of quantum dots. Toxicol. Appl. Pharmacol. 2009, 238, 280-288.

7 Cho, S. J.; Maysinger, D.; Jain, M.; Roder, B.; Hackbarth, S.; Winnik, F. M. Long-term exposure to CdTe quantum dots causes functional impairments in live cells. Langmuir 2007, 23, 1974-1980.

8 Hoshino, A.; Hanada, S.; Yamamoto, K. Toxicity of nanocrystal quantum dots: the relevance of surface modifications. Arch. Toxicol. 2011, 85, 707-720.

9 Hauck, T. S.; Anderson, R. E.; Fischer, H. C.; Newbigging, S.; Chan, W. C. W. In vivo quantum-dot toxicity assessment. Small 2010, 6, 138-144.

10 Zhu, Z.-J.; Yeh, Y.-C.; Tang, R.; Yan, B.; Tamayo, J.; Vachet, R. W.; Rotello, V. M. Stability of quantum dots in live cells. Nat. Chem. 2011, 3, 963-968.

11 Halliwell, B.; Gutteridge, J. M. C. Free radicals in biology and medicine, 3rd ed.; Oxford University Press: New York, 1999.

12 Meng, H.; Xia, T.; George, S.; Nel, A. E. A predictive toxicological paradigm for the safety assessment of nanomaterials. ACS Nano 2009, 3, 1620-1627.

13 Davidson, S. M.; Duchen, M. R. Endothelial mitochondria: contributing to vascular function and disease. Circ. Res. 2007, 100, 1128-1141.

14 Yan, M.; Zhang, Y.; Xu, K. D.; Fu, T.; Qin, H. Y.; Zheng, X. X. An in vitro study of vascular endothelial toxicity of CdTe quantum dots. Toxicology 2011, 282, 94-103.

15 Zhang, L. W.; Monteiro-Riviere, N. A. Assessment of quantum dot penetration into intact, tape-stripped, abraded and flexed rat skin. Skin Pharmacol. Phys. 2008, 21, 166-180.

16 Mortensen, L. J.; Oberdorster, G.; Pentland, A. P.; Delouise, L. A. In vivo skin penetration of quantum dot nanoparticles in the murine model: the effect of UVR. Nano Lett. 2008, 8, 2779-2787.

17 Romoser, A. A.; Chen, P. L.; Berg, J. M.; Seabury, C.; Ivanov, I.; Criscitiello, M. F.; Sayes, C. M. Quantum dots trigger immunomodulation of the NF kappa B pathway in human skin cells. Mol. Immunol. 2011, 48, 1349-1359.

18 Kuo, T. R.; Lee, C. F.; Lin, S. J.; Dong, C. Y.; Chen, C. C.; Tan, H. Y. Studies of Intracorneal Distribution and Cytotoxicity of Quantum Dots: Risk Assessment of Eye Exposure. Chem. Res. Toxicol. 2011, 24, 253-261.

19 Maysinger, D.; Behrendt, M.; Lalancette-Herbert, M.; Kriz, J. Real-time imaging of astrocyte response to quantum dots: In vivo screening model system for biocompatibility of nanoparticles. Nano Lett. 2007, 7, 2513-2520.

20 Lalancette-Hebert, M.; Moquin, A.; Choi, A. 0.; Kriz, J.; Maysinger, D. LipopolysaccharideQD micelles induce marked induction of TLR2 and lipid droplet accumulation in olfactory bulb microglia. Mol. Pharmaceutics 2010, 7, 1183-1194.

21 Tang, M.; Li, Z.; Chen, L.; Xing, T.; Hu, Y.; Yang, B.; Ruan, D. Y.; Sun, F.; Wang, M. The effect of quantum dots on synaptic transmission and plasticity in the hippocampal dentate gyrus area of anesthetized rats. Biomaterials 2009, 30, 4948-4955.

22 Tang, M.; Wang, M.; Xing, T.; Zeng, J.; Wang, H.; Ruan, D. Y. Mechanisms of unmodified CdSe quantum dot-induced elevation of cytoplasmic calcium levels in primary cultures of rat hippocampal neurons. Biomaterials 2008, 29, 4383-4391.

23 Gosso, S.; Gavello, D.; Giachello, C. N. G.; Franchino, C.; Carbone, E.; Carabelli, V. The effect of CdSe-ZnS quantum dots on calcium currents and catecholamine secretion in mouse chromaffin cells. Biomaterials 2011, 32, 9040-9050.

24 Tarantola, M.; Schneider, D.; Sunnick, E.; Adam, H.; Pierrat, S.; Rosman, C.; Breus, V.; Sonnichsen, C.; Basche, T.; Wegener, J.; Janshoff, A. Cytotoxicity of metal and semiconductor nanoparticles indicated by cellular micromotility. ACS Nano 2009, 3, 213-222.

25 Darbre, P. D. Metalloestrogens: an emerging class of inorganic xenoestrogens with potential to add to the oestrogenic burden of the human breast. J. Appl. Toxicol. 2006, 26, 191-197.

26 Jain, M. P.; Vaisheva, F.; Maysinger, D. Metalloestrogenic effects of quantum dots. Nanomedicine 2012, 7, 23-37.

27 Singh, N.; Manshian, B.; Jenkins, G. J.; Griffiths, S. M.; Williams, P. M.; Maffeis, T. G.; Wright, C. J.; Doak, S. H. NanoGenotoxicology: the DNA damaging potential of engineered nanomaterials. Biomaterials 2009, 30, 3891-3914.

28 Anas, A.; Akita, H.; Harashima, H.; Itoh, T.; Ishikawa, M.; Bju, V. Photosensitized breakage and damage of DNA by CdSe-ZnS quantum dots. J. Phys. Chem. B 2008, 112, 10005-10011.

29 Green, M.; Howman, E. Semiconductor quantum dots and free radical induced DNA nicking. Chem. Commun. 2005, 121-123.

30 Hoshino, A.; Fujioka, K.; Oku, T.; Suga, M.; Sasaki, Y. F.; Ohta, T.; Yasuhara, M.; Suzuki, K.; Yamamoto, K. Physicochemical properties and cellular toxicity of nanocrystal quantum dots depend on their surface modification. Nano Lett. 2004, 4, 2163-2169.

31 Gagné, F.; Fortier, M.; Yu, L.; Osachoff, H. L.; Skirrow, R. C.; van Aggelen, G.; Gagnon, C.; Fournier, M. Immunocompetence and alterations in hepatic gene expression in rainbow trout exposed to CdS/CdTe quantum dots. J. Environ. Monitor. 2010, 12, 1556-1565.

32 Callinan, P. A.; Feinberg, A. P. The emerging science of epigenomics. Hum. Mol. Genet. 2006, 15, R95-R101. 
33 Esteller, M. The necessity of a human epigenome project. Carcinogenesis 2006, 27, 1121-1125.

34 Choi, A. 0.; Brown, S. E.; Szyf, M.; Maysinger, D. Quantum dot-induced epigenetic and genotoxic changes in human breast cancer cells. J. Mol. Med. 2008, 86, 291-302.

35 Gaponik, N.; Rogach, A. L. Thiol-capped CdTe nanocrystals: progress and perspectives of the related research fields. Phys. Chem. Chem. Phys. 2010, 12, 8685-8693.

36 Gagné, F.; Auclair, J.; Turcotte, P.; Fournier, M.; Gagnon, C.; Sauve, S.; Blaise, C. Ecotoxicity of CdTe quantum dots to freshwater mussels: impacts on immune system, oxidative stress and genotoxicity. Aquat. Toxicol. 2008, 86, 333-340.

37 Wang, Q.; Fang, T.; Liu, P.; Min, X.; Li, X. Study of the bioeffects of CdTe quantum dots on Escherichia coli cells. J. Colloid Interface Sci. 2011, 363, 476-480.

38 Priester, J. H.; Stoimenov, P. K.; Mielke, R. E.; Webb, S. M.; Ehrhardt, C.; Zhang, J. P.; Stucky, G. D.; Holden, P. A. Effects of soluble cadmium salts versus CdSe quantum dots on the growth of planktonic Pseudomonas aeruginosa. Environ. Sci. Technol. 2009, 43, 2589-2594.

39 Werlin, R.; Priester, J. H.; Mielke, R. E.; Kramer, S.; Jackson, S.; Stoimenov, P. K.; Stucky, G. D.; Cherr, G. N.; Orias, E.; Holden, P. A. Biomagnification of cadmium selenide quantum dots in a simple experimental microbial food chain. Nat. Nanotechnol. 2011, 6, 65-71.

40 Chu, M. Q.; Wu, Q.; Yang, H.; Yuan, R. Q.; Hou, S. K.; Yang, Y. F.; Zou, Y. J.; Xu, S.; Xu, K. Y.; Ji, A. L.; Sheng, L. Y. Transfer of quantum dots from pregnant mice to pups across the placental barrier. Small 2010, 6, 670-678.

41 Baker, S. N.; Baker, G. A. Luminescent carbon nanodots: Emergent nanolights. Angew. Chem., Int. Ed. 2010, 49, 6726-6744.

42 Peng, J.; Gao, W.; Gupta, B. K.; Liu, Z.; Romero-Aburto, R.; Ge, L.; Song, L.; Alemany, L. B.; Zhan, Z.; Gao, G.; Vithayathil, S. A.; Kaipparettu, B. A.; Marti, A. A.; Hayashi, T.; Zhu, J.-J.;
Ajayan, P. M. Graphene quantum dots derived from carbon fibers. Nano Lett. 2012, 12 , 844-849.

43 Shen, J.; Zhu, Y.; Yang, X.; Li, C. Graphene quantum dots: Emergent nanolights for bioimaging, sensors, catalysis and photovoltaic devices. Chem. Commun. 2012, 48, 3686-3699.

44 Ray, S. C.; Saha, A.; Jana, N. R.; Sarkar, R. Fluorescent carbon nanoparticles: Synthesis, characterization, and bioimaging application. J. Phys. Chem. C 2009, 113, 18546-18551.

45 Sun, X.; Liu, Z.; Welsher, K.; Robinson, J. T.; Goodwin, A.; Zaric, S.; Dai, H. Nano-graphene oxide for cellular imaging and drug delivery. Nano Res. 2008, 1, 203-212.

46 Bimbo, L. M.; Sarparanta, M.; Santos, H. A.; Airaksinen, A. J.; Makila, E.; Laaksonen, T.; Peltonen, L.; Lehto, V. P.; Hirvonen, J.; Salonen, J. Biocompatibility of thermally hydrocarbonized porous silicon nanoparticles and their biodistribution in rats. ACS Nano 2010, 4, 3023-3032.

47 Henderson, E. J.; Shuhendler, A. J.; Prasad, P.; Baumann, V.; Maier-Flaig, F.; Faulkner, D. 0.; Lemmer, U.; Wu, X. Y.; Ozin, G. A. Colloidally stable silicon nanocrystals with nearinfrared photoluminescence for biological fluorescence imaging. Small 2011, 7, 2507-2516.

48 Erogbogbo, F.; Yong, K.-T.; Hu, R.; Law, W.-C.; Ding, H.; Chang, C.-W.; Prasad, P. N.; Swihart, M. T. Biocompatible magnetofluorescent probes: Luminescent silicon quantum dots coupled with superparamagnetic iron(III) oxide. ACS Nano 2010, 4, 5131-5138.

49 Dhawan, A.; Sharma, V. Toxicity assessment of nanomaterials: Methods and challenges. Anal. Bioanal. Chem. 2010, 398, 589-605.

50 Warheit, D. B. How meaningful are the results of nanotoxicity studies in the absence of adequate material characterization? Toxicol. Sci. 2008, 101, 183-185. 